\title{
Transverse Momentum in Semi-Inclusive DIS
}

\author{
Alessandro Bacchetta
}

Theory Group, DESY, 22603 Hamburg, Germany

\begin{abstract}
The cross section for semi-inclusive deep inelastic scattering can be decomposed in terms of 18 structure functions. At low transverse momentum of the detected hadron, the structure functions can be expressed in terms of transverse-momentum-dependent parton distribution and fragmentation functions. Here, a few selected examples are illustrated and discussed.
\end{abstract}

I present a selection of results from a recent work [2], where semi-inclusive deep inelastic scattering, $\ell(l)+N(P) \rightarrow \ell(l-q)+h\left(P_{h}\right)+X$, was analyzed in the regime of low transverse momentum of the detected hadron, with the goal of completing the existing literature on the subject. In the following, the standard variables $Q^{2}, x, y, z$ are used. $M$ and $M_{h}$ denote the respective masses of the nucleon and of the hadron $h$. Azimuthal angles are defined in accordance to the Trento conventions [3]. $P_{h \perp}$ denotes the component of $P_{h}$ perpendicular to $q$, in, e.g., the proton-photon Breit frame.

Assuming single photon exchange, the lepton-hadron cross section can be computed as the contraction of the hadronic and the leptonic tensor and expressed by a set of structure functions. For unpolarized beam and target, the cross section contains only four structure functions. Neglecting corrections of order $1 / Q^{2}$, it can be written as

$$
\begin{aligned}
\frac{d \sigma}{d \Gamma}= & \frac{2 \pi \alpha^{2} y}{8 z Q^{4}} 2 M W^{\mu \nu} L_{\mu \nu}=\frac{2 \pi \alpha^{2}}{x y Q^{2}}\left\{\left(1-y+y^{2} / 2\right) F_{U U, T}+(1-y) F_{U U, L}\right. \\
& \left.+(2-y) \sqrt{1-y} \cos \phi_{h} F_{U U}^{\cos \phi_{h}}+(1-y) \cos \left(2 \phi_{h}\right) F_{U U}^{\cos 2 \phi_{h}}\right\},
\end{aligned}
$$

where $d \Gamma=d x d y d z d \phi_{h} d P_{h \perp}^{2}$. The structure functions depend on $x, Q^{2}, z$ and $P_{h \perp}^{2}$. There is no established notation for the structure functions. Here as in [2], the superscript indicates the azimuthal modulation generated in the cross section. The first and second subscript indicate the respective polarization of beam and target. When needed, the third subscript specifies the polarization of the virtual photon.

In the kinematical limit where $Q^{2}$ becomes large while $x, z$ and $P_{h \perp}^{2}$ remain fixed, it has been proven [4] that the cross section up to leading order in $1 / Q$ can be factorized into a hard photon-quark scattering process and transverse momentum dependent distribution and fragmentation functions (for the regime $M^{2} \ll P_{h \perp}^{2} \ll Q^{2}$, see e.g. [5]). Here, it is assumed that factorization applies up to subleading order in the $1 / Q$ expansion, and only graphs with the hard scattering at tree level are considered. Loops can then only occur as shown, e.g., in Fig. 1 b, c. It is convenient to introduce the shorthand notations

$$
\hat{\mathbf{h}}=\mathbf{P}_{h \perp} /\left|\mathbf{P}_{h \perp}\right| \quad \text { and } \quad \mathcal{C}[\ldots]=x \sum_{a} e_{a}^{2} \int d^{2} \mathbf{p}_{T} d^{2} \mathbf{k}_{T} \delta^{(2)}\left(\mathbf{p}_{T}-\mathbf{k}_{T}-\mathbf{P}_{h \perp} / z\right) \ldots
$$

where the sum runs over the quark and antiquark flavors $a$, and $e_{a}$ denotes their fractional 


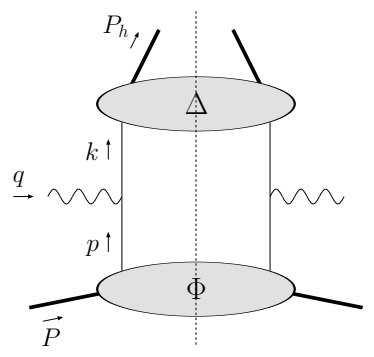

(a)

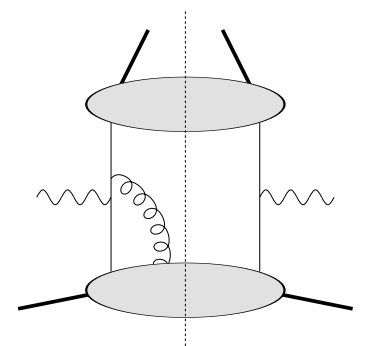

(b)

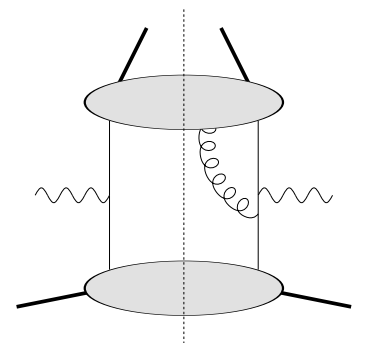

(c)

Figure 1: Examples of graphs contributing to semi-inclusive DIS at low transverse momentum of the produced hadron.

charge. The corresponding expression of the hadronic tensor is [6]

$$
\begin{aligned}
2 M W^{\mu \nu} & =\frac{2 z}{x} \mathcal{C}\left\{\operatorname{Tr}\left[\Phi^{a}\left(x, p_{T}\right) \gamma^{\mu} \Delta^{a}\left(z, k_{T}\right) \gamma^{\nu}\right]-\frac{1}{Q \sqrt{2}} \operatorname{Tr}\left[\gamma^{\alpha} \gamma^{-} \gamma^{\nu} \tilde{\Phi}_{A \alpha}^{a}\left(x, p_{T}\right)\right.\right. \\
& \left.\left.\times \gamma^{\mu} \Delta^{a}\left(z, k_{T}\right)+\gamma^{\alpha} \gamma^{+} \gamma^{\mu} \tilde{\Delta}_{A \alpha}^{a}\left(z, k_{T}\right) \gamma^{\nu} \Phi^{a}\left(x, p_{T}\right)+\text { h.c. }\right]\right\}+\mathcal{O}\left(1 / Q^{2}\right) .
\end{aligned}
$$

The quark-quark correlators $\Phi$ and $\Delta$ can be decomposed up to order $1 / Q$ in a general way in terms of transverse-momentum-dependent distribution functions. The quark-gluonquark correlators $\tilde{\Phi}_{A}$ and $\tilde{\Delta}_{A}$ can be related to the quark-quark correlators by means of the QCD equations of motion. Eventually, this allows the computation of the hadronic tensor and of the structure functions appearing in the cross section.

The full calculation leads to the following results [2]

$$
\begin{aligned}
F_{U U, T} & =\mathcal{C}\left[f_{1} D_{1}\right], \quad F_{U U, L}=0, \\
F_{U U}^{\cos \phi_{h}} & =\frac{2 M}{Q} \mathcal{C}\left[-\frac{\hat{\mathbf{h}} \cdot \mathbf{k}_{T}}{M_{h}}\left(x h H_{1}^{\perp}+\frac{M_{h}}{M} f_{1} \frac{\tilde{D}^{\perp}}{z}\right)-\frac{\hat{\mathbf{h}} \cdot \mathbf{p}_{T}}{M}\left(x f^{\perp} D_{1}+\frac{M_{h}}{M} h_{1}^{\perp} \frac{\tilde{H}}{z}\right)\right], \\
F_{U U}^{\cos 2 \phi_{h}} & =\mathcal{C}\left[-\frac{2\left(\hat{\mathbf{h}} \cdot \mathbf{k}_{T}\right)\left(\hat{\mathbf{h}} \cdot \mathbf{p}_{T}\right)-\mathbf{k}_{T} \cdot \mathbf{p}_{T}}{M M_{h}} h_{1}^{\perp} H_{1}^{\perp}\right] .
\end{aligned}
$$

The distribution (fragmentation) functions $f_{1}\left(D_{1}\right), f^{\perp}\left(\tilde{D}^{\perp}\right), h(\tilde{H})$, and $h_{1}^{\perp}\left(H_{1}^{\perp}\right)$ depend on $x(z)$ and $p_{T}^{2}\left(k_{T}^{2}\right)$ and should carry a flavor index $a$.

To order $1 / Q$, the function $F_{U U, L}$ vanishes. The structure function $F_{U U}^{\cos \phi_{h}}$ is subleadingtwist, i.e. $1 / Q$-suppressed compared to the other two nonvanishing ones. Measurements of $F_{U U}^{\cos \phi_{h}}$ have been reported in Refs. [7, 8]. This structure function is associated with the so-called Cahn effect. If one neglects the contribution from interaction-dependent functions and T-odd functions Eq. (5) becomes

$$
F_{U U}^{\cos \phi_{h}} \approx \frac{2 M}{Q} \mathcal{C}\left[-\frac{\hat{\mathbf{h}} \cdot \mathbf{p}_{T}}{M} f_{1} D_{1}\right] .
$$

This coincides with the $\cos \phi_{h}$ term calculated to order $1 / Q$ in the parton model with intrinsic transverse momentum included in distribution and fragmentation functions, see e.g. Eqs. (32) and (33) in Ref. [9]. 
The structure function $F_{U U}^{\cos 2 \phi_{h}}$ contains the functions $h_{1}^{\perp}$ (Boer-Mulders function) and $H_{1}^{\perp}$ (Collins function). Measurements have been reported in Refs. [8].

Upon integration over the transverse momentum of the outgoing hadron, the integrated semi-inclusive DIS result is recovered, i.e.,

$$
F_{U U, T}\left(x, z, Q^{2}\right)=x \sum_{a} e_{a}^{2} f_{1}^{a}(x) D_{1}^{a}(z), \quad F_{U U, L}\left(x, z, Q^{2}\right)=0,
$$

where

$$
f_{1}^{a}(x)=\int d^{2} \mathbf{p}_{T} f_{1}^{a}\left(x, p_{T}^{2}\right), \quad D_{1}^{a}(z)=z^{2} \int d^{2} \mathbf{k}_{T} D_{1}^{a}\left(z, k_{T}^{2}\right)
$$

Finally, the standard inclusive DIS structure function is obtained by

$$
F_{T}\left(x, Q^{2}\right)=\int d z z F_{U U, T}\left(x, z, Q^{2}\right)=x \sum_{a} e_{a}^{2} f_{1}^{a}(x) .
$$

The analysis of the cross section and of the structure functions for polarized beams and targets can be carried out in an analogous way. There are in total 18 structure functions, eight are $\mathcal{O}\left(1 / Q^{0}\right)$, eight are $\mathcal{O}(1 / Q)$, and two are of higher order. One subleading-twist structure function, $F_{L U}^{\sin \phi_{h}}$, appears if the beam is longitudinally polarized and the target unpolarized. The only available measurement is by the CLAS collaboration [10]. Two structure functions, $F_{U L}^{\sin \phi_{h}}$ and $F_{U L}^{\sin 2 \phi_{h}}$, appear if the beam is unpolarized and the target longitudinally polarized. They have been measured only by the HERMES collaboration [11]. Two structure functions, $F_{L L}$ and $F_{L L}^{\cos \phi_{h}}$, require longitudinal polarization of both beam and target. The first one has already been measured in the past, but little is known about its transverse-momentum dependence, which could yield interesting information on orbital angular momentum.

The structure functions with transversely polarized targets are nine in total. $F_{U T, T}^{\sin \left(\phi_{h}-\phi_{S}\right)}$ and $F_{U T}^{\sin \left(\phi_{h}+\phi_{S}\right)}$ received a lot of attention from the theoretical and experimental side in the last years. They contain the Sivers and transversity distribution functions, respectively [12]. The COMPASS collaboration has recently completed the task of measuring eight of these structure functions for a deuteron target; preliminary data were presented during this conference [13]. I highlight here two illustrative examples that deserve particular attention. The first one is

$$
F_{L T}^{\cos \left(\phi_{h}-\phi_{S}\right)}=\mathcal{C}\left[\frac{\hat{\mathbf{h}} \cdot \mathbf{p}_{T}}{M} g_{1 T} D_{1}\right],
$$

requiring both longitudinally polarized beam and transversely polarized target. Through the measurement of this asymmetry and with the knowledge of the unpolarized fragmentation functions, it is possible to measure the parton distribution function $g_{1 T}^{q}$, which is the only chiral-even, T-even, leading-twist function in addition to the well-known unpolarized distribution function, $f_{1}^{q}$, and helicity distribution function, $g_{1}^{q}$.

Another interesting structure function is

$$
\begin{aligned}
F_{U T}^{\sin \phi_{S}}=\frac{2 M}{Q} \mathcal{C}\left\{\left(x f_{T} D_{1}-\frac{M_{h}}{M} h_{1} \frac{\tilde{H}}{z}\right)\right. \\
\left.\quad-\frac{\mathbf{k}_{T} \cdot \mathbf{p}_{T}}{2 M M_{h}}\left[\left(x h_{T} H_{1}^{\perp}+\frac{M_{h}}{M} g_{1 T} \frac{\tilde{G}^{\perp}}{z}\right)-\left(x h_{T}^{\perp} H_{1}^{\perp}-\frac{M_{h}}{M} f_{1 T}^{\perp} \frac{\tilde{D}^{\perp}}{z}\right)\right]\right\},
\end{aligned}
$$


generating a $\sin \phi_{S}$ modulation in single-spin asymmetries with a transversely polarized target. Upon integration over the transverse momentum of the outgoing hadron, only one term survives, namely

$$
F_{U T}^{\sin \phi_{S}}\left(x, z, Q^{2}\right)=-x \sum_{a} e_{a}^{2} \frac{2 M_{h}}{Q} h_{1}^{a}(x) \frac{\tilde{H}^{a}(z)}{z},
$$

where the transversity distribution function appears, multiplied by a subleading-twist fragmentation function. It could therefore be a good observable for transversity studies.

Another interesting limit of Eq. (12) is that of semi-inclusive jet production, $\ell(l)+$ $N(P) \rightarrow \ell(l-q)+\operatorname{jet}\left(P_{j}\right)+X$. The structure function for this process can be obtained from Eq. (12) by replacing $D_{1}\left(z, k_{T}^{2}\right)$ with $\delta(1-z) \delta^{(2)}\left(\mathbf{k}_{T}\right)$, setting all other fragmentation functions to zero and integrating over $z$. This gives

$$
F_{U T}^{\sin \phi_{S}}\left(x, P_{j \perp}^{2}, Q^{2}\right)=x \sum_{a} e_{a}^{2} \frac{2 M}{Q} x f_{T}^{a}\left(x, P_{j \perp}^{2}\right),
$$

In totally inclusive DIS this structure function has to vanish due to time-reversal invariance. Starting from Eq. (13) or Eq. (14) and performing the required integrations, the following two relations can be derived

$$
\sum_{h} \int d z \tilde{H}^{a}(z)=0, \quad \quad \int d^{2} \mathbf{p}_{T} f_{T}\left(x, p_{T}^{2}\right)=0 .
$$

The second relation can also be used to establish a connection between the Sivers function and the Qiu-Sterman effect (see, e.g., [14]).

In conclusion, I presented a selection of the results obtained in [2], where the cross section for semi inclusive deep inelastic scattering off a polarized nucleon for low transverse momentum of the detected hadron was analyzed, completing the existing literature. I pointed out that there are in general 18 structure functions, nine of which have been already measured in some experiments. More measurements will hopefully come in the future from HERMES, COMPASS, and JLab.

\section{References}

[1] Slides:

http://indico. cern. ch/contributionDisplay $\cdot$ py? contribId=163\&sessionId=4\&conf Id=9499

[2] A. Bacchetta et al., JHEP 02, 093 (2007).

[3] A. Bacchetta, U. D'Alesio, M. Diehl and C. A. Miller, Phys. Rev. D70, 117504 (2004).

[4] X. Ji, J.-P. Ma and F. Yuan, Phys. Rev. D71, 034005 (2005).

[5] Y. Koike and K. Tanaka, these proceedings, arXiv:0706.2545 [hep-ph], arXiv:0706.2117 [hep-ph].

[6] D. Boer, P. J. Mulders and F. Pijlman, Nucl. Phys. B667, 201 (2003).

[7] EMC, M. Arneodo et al., Z. Phys. C34, 277 (1987). M. R. Adams, et al., Phys. Rev. D48, 5057 (1993),

[8] ZEUS, J. Breitweg et al., Phys. Lett. B481, 199 (2000). S. Chekanov, et al., hep-ex/0608053.

[9] M. Anselmino et al., Phys. Rev. D71, 074006 (2005).

[10] CLAS, H. Avakian et al., Phys. Rev. D69, 112004 (2004).

[11] HERMES, A. Airapetian et al., Phys. Lett. B622, 14 (2005). Phys. Lett. B562, 182-192 (2003).

[12] A. Bressan, M. Diefenthaler, these proceedings.

[13] A. Kotzinian, these proceedings, arXiv:0705.2402 [hep-ex].

[14] A. Bacchetta, hep-ph/0511085. 